\title{
Analyzing Vaccination Priority Judgments for 132 Occupations Using Word Vector Models
}

\author{
Atsushi Ueshima* \\ Graduate School of Arts and Letters, Tohoku University \\ Sendai, Japan; Japan Society for the Promotion of Science \\ Tokyo, Japan \\ ueshima73@gmail.com
}

\begin{abstract}
Most human societies conduct a high degree of division of labor based on occupation. However, determining the occupational field that should be allocated a scarce resource such as vaccine is a topic of debate, especially considering the COVID-19 situation. Though it is crucial that we understand and anticipate people's judgments on resource allocation prioritization, quantifying the concept of occupation is a difficult task. In this study, we investigated how well people's judgments on vaccination prioritization for different occupations could be modeled by quantifying their knowledge representation of occupations as word vectors in a vector space. The results showed that the model that quantified occupations as word vectors indicated high out-of-sample prediction accuracy, enabling us to explore the psychological dimension underlying the participants' judgments. These results indicated that using word vectors for modeling human judgments about everyday concepts allowed prediction of performance and understanding of judgment mechanisms
\end{abstract}

\section{CCS CONCEPTS}

- CCS; • Applied computing; • Law, social and behavioral sciences; $\bullet$ Psychology;

\section{KEYWORDS}

Judgment and Decision Making, Word Embedding, Distributive Justice, Meanings, Scare Resource Allocation

\section{ACM Reference Format:}

Atsushi Ueshima and Hiroki Takikawa. 2021. Analyzing Vaccination Priority Judgments for 132 Occupations Using Word Vector Models. In IEEE/WIC/ACM International Conference on Web Intelligence (WI-IAT '21 Companion), December 14-17, 2021, ESSENDON, VIC, Australia. ACM, New York, NY, USA, 7 pages. https://doi.org/10.1145/3498851.3498933

*Place the footnote text for the author (if applicable) here.

Permission to make digital or hard copies of all or part of this work for personal or classroom use is granted without fee provided that copies are not made or distributed for profit or commercial advantage and that copies bear this notice and the full citation on the first page. Copyrights for components of this work owned by others than the author(s) must be honored. Abstracting with credit is permitted. To copy otherwise, or republish, to post on servers or to redistribute to lists, requires prior specific permission and/or a fee. Request permissions from permissions@acm.org.

WI-IAT '21 Companion, December 14-17, 2021, ESSENDON, VIC, Australia

(c) 2021 Copyright held by the owner/author(s). Publication rights licensed to ACM

ACM ISBN 978-1-4503-9187-0/21/12 . \$ $\$ 15.00$

https://doi.org/10.1145/3498851.3498933

\author{
Hiroki Takikawa \\ Graduate School of Arts and Letters Tohoku University \\ Sendai, Japan \\ hiroki.takikawa.d3@tohoku.ac.jp
}

\section{INTRODUCTION}

Human societies consist of a high degree of division of labor based on occupation. Currently, due to the COVID-19 pandemic, just allocation of a scarce resource such as vaccines has become a topic of intense debate. Distributive justice, especially during this pandemic, has captured attention worldwide and is universally essential as human society continues to operate with finite resources. Therefore, understanding and predicting people's judgment in prioritizing resource allocation across occupations is critical. This study attempted to predict and understand people's judgments when allocating scarce resources to people in various occupations. To achieve this goal, we used a vector space semantic model, which enabled us to quantify the meanings of various occupations as numeric word vectors based on a large corpus.

Previous research on resource allocation has shown that several types of distributional preferences exist regarding resource allocation. For example, studies in experimental economics, behavioral economics, and social psychology have shown that the primary factors influencing distributive judgments include inequality $[1,8]$, the welfare of the worst-off $[7,9,13]$, and social efficiency (the welfare of society as a whole [6]) of resource distribution. In addition, studies in the field of political science have underscored people's attitudes towards resource (re)distribution, using large and representative samples [2, 3, 17].

These studies are crucial for understanding people's distributional judgments and decision-making. However, the literature is not sufficient for understanding people's distributional judgments involving a natural concept like occupation. One of the reasons behind this limitation is that it has been challenging to quantify everyday concepts in modeling distributional judgments. This is not to say that the literature does not quantify occupations. For example, the occupational prestige score quantifies occupations. Moreover, a database such as the Occupational Information Network $\left(\mathrm{O}^{*} \mathrm{NET}\right)$ provides quantitative scores of various occupations in terms of necessary skills, abilities, and work contexts.

Though insightful, these scores usually reflect only a tiny part of the impressions and meanings people have about occupations. Hence, they are not entirely suitable if we intend to predict people's distributional judgments regarding various occupations with high out-of-sample accuracy rates, which requires rich knowledge representations of occupations. To model the distributional judgments regarding various occupations, it is necessary to quantify people's impressions of occupations as comprehensively as possible.

Recent research in cognitive science has successfully employed vector space semantic models to map various judgments about everyday concepts $[4,20,28]$. Vector space semantic models exploit 
the distributional pattern of words in a large corpus to obtain a vector representation of each word. For example, in the sentence "Farmers have political influence," the word "Farmers" appears in a political context. If such sentences in which farmers appear in a political context are frequent in a corpus, the word "farmer" has a vector representation similar to that of other political words. In other words, the meaning of a word depends on the context in which the word appears (distributional hypothesis [10]). Thus, the distributional hypothesis implies that words that occur in similar contexts have similar meanings. Then, based on the distributional hypothesis, it is possible to represent the meanings of words as numeric vectors. We can use such vector representations of words to quantify people's knowledge representation and meaning in words or concepts $[15,20]$. We assume that distributional hypothesis would imply the quantification of meaning of people's understanding towards knowledge because text corpora can reflect people's language experience, and that can shape people's semantic judgments [28]. The current study used this vector space semantic model to quantify people's rich knowledge representation of various occupations in numeric word vectors. We can then feed the vector representations of each occupation into the model as an explanatory variable.

Taking advantage of the fact that the knowledge representation for each occupation can be quantified as a word vector, this study attempted to predict vaccination priority judgment for more than 130 different occupations and understand its psychological basis. Predicting the kind of resource distribution that can be supported may help formulate relevant social policies. Notably, we can obtain word vectors of occupations from a corpus that already exists. Hence, using word vectors as input variables can enable the modeling of people's judgments rapidly and inexpensively.

As discussed later, in this study, we used word vectors in the framework of multiple regression analysis rather than using prediction-specialized methods such as deep neural networks. Using a simpler and more interpretable model, we attempted to understand the psychological underpinnings of people's judgments.

Regarding the out-of-sample predictive accuracy of the model using the word vector representation of occupations, this study made two predictions. First, we predicted that the mean observed rating for vaccination priority of each occupation (measured on a Likert scale ranging from 0 to 4 ) would highly correlate with the model's mean predicted rating. Since correlation coefficients are generally considered high when they are above 0.5 , this study set the heuristic that if the out-of-sample correlation between the observed values and the model predicted values were above 0.5 , the model's prediction accuracy would be adequate. Accordingly, we predicted that a model using word vectors could achieve this criterion. We acknowledge that a model's prediction accuracy sufficiently depends on how we use the model in the real world. However, we adopted the above criterion because the model evaluation based on real-world applications was not suitable for the current study. Second, we evaluated the predictive performance of the word vector model by comparing it with a benchmark model. Then, we predicted that the model using word vectors would show a higher predictive performance than the benchmark model. Although there are several ways to set up a benchmark model, we used a multiple regression model with three explanatory variables as the benchmark for model comparison. As explanatory variables, we asked participants to respond to three items that were highly related to vaccination priority judgments. As described in the methods section, we specifically asked each participant three questions governed by: (1) social importance, (2) personal importance, and (3) familiarity with each occupation. The participants answered these questions immediately after completing the vaccine distribution priority rating for each occupation. We compared the two models using the widely applicable information criterion (WAIC).

\section{METHODS}

\subsection{Participants and procedures}

We recruited 250 participants through a Japanese crowdsourcing website. Excluding 7 participants for whom complete data were not available due to communication errors or inattention, our analyses used 243 participants in total (136 women, 105 men, and two preferred not to specify; mean age $=40.88$ years, $S D=9.86$ ). Informed consent was obtained from each participant before the study, using a form approved by the ethics committee of the Graduate School of Arts and Letters, Tohoku University. The participants responded using their personal computers. Participants answered the question "For the following five occupations, if certain occupations are given priority for vaccination against novel coronavirus infections, to what extent do you think the prioritization is justified?" by selecting responses on a 5-point Likert scale ranging from 0 (Should be given the lowest priority) to 4 (Should be given the highest priority). The five occupations presented were randomly selected for each participant from the 132 occupations shown in Table 1. Before answering this vaccination priority question, participants completed a questionnaire that encouraged them to think specifically about each of the five occupations (four items: average annual income, percentage of female workers, average age of workers, and the number of acquaintances in each occupation) and played an economic game, which we have included in a different study. At the end of the questionnaire, participants provided demography-related details, such as their age, gender, and occupation. Participants received 300 yen (approximately US\$3) for participation.

\subsection{Models and evaluation metric}

As explained above, we examined how well a model using vector representations of each occupation could predict the vaccination priority judgment for various occupations. We obtained vector representations of each occupation from a publicly available pretrained word2vec resource, chiVe [14]. It is one of the most extensive and publicly available Japanese pre-trained word2vec resources and uses a high-quality corpus of the National Institute for Japanese Language and Linguistics, which contains approximately 100 million web pages as a training corpus. Based on previous research $[4,28]$, we modeled the participants' vaccine priority judgment $y_{i}$ against occupation $x_{i}$ as follows.

$$
\begin{gathered}
y_{i}=\alpha_{\text {participant }[i]}+w \boldsymbol{x}_{\boldsymbol{i}}+\varepsilon_{i}, \\
\alpha_{\text {participant }[i]}=\alpha_{\text {population }}+\eta_{\text {participant }[i]}
\end{gathered}
$$

Here, $x_{i}$ is the 300 -dimensional vector representing occupation $i$ obtained from the pre-trained word2vec resource and an explanatory 
Table 1: A list of 132 occupations used in the study. For each participant, we presented five occupations randomly chosen out of 132 occupations as judgment targets. We selected the 132 occupations using the Japanese standard occupational classification (2009) and the Japanese version of Wikipedia's entry on "occupation (shokugyo ichiran)" as reference. For corresponding Japanese words, see Table S1 provided in https://osf.io/mgpws/?view_only=16acfb4e385e4729b1239c8b8c60ddef

\begin{tabular}{|c|c|c|c|c|c|c|c|c|}
\hline Scientist & Nursery teacher & Monk & Typist & |Tour conductor & |Fireman & Fiber Spinning & Transportation & Gas station \\
\hline Farmer & Counselor & Dancer & Call center & Room attendant & BodyEuard & Embroidery & Home delivery & Fish retailer \\
\hline Fisherman & Painter (artist) & Stand-up comedian & Market research & Bus guide & Security guard & Welding & Shippine & Shoe store \\
\hline Dairy farmer & Photographer & Potter & Real estate agent & Bartender & Guard & Power plant & House cleaning & dothing store \\
\hline Idol & Career consultant & Librarian & Advertising agency & Cabaret woman & Grower & Navigator & Dishwasher & Butcher \\
\hline Illustrator & Nutritionist & Announcer & Pawnbroker & Fashion model & Landkcaper & Tow truck & Martial artist & Singer \\
\hline Animator & Architect & Actor & Landlord & Flight attendant & Henter & One-man bus & YouTuber & Stylist \\
\hline Cartoonist & Copywriter & Lawyer & Homeowner & Babysitter & Gardener & Engineer & Comedian & Railroad worker \\
\hline Graphic designer & Bonesetter & Pastor & Fortune teller & Hous ekeeper & Diver & Carpenter & Baker & Sailor \\
\hline Doctor & Interpreter & Pharmacist & Caregiver & Barber & House painter & Miner & Florist & Writer \\
\hline Programmer & Translator & Care manager & Dry cleaner & Waiter & Mechanic & Steel worker & Grocery store & Teacher \\
\hline Nowelist & Shinto priest & Midwife & Janitor & Funeral director & Pyrotechnician & Wall painter & Blogger & Construction worker \\
\hline Pianist & Stuntman & Reporter & Hairdresser & Hostess & Pig iron maker & Tatami-maker & College student & \\
\hline Accountant & Voice actor & Bank derk & Therapist & Cook & Blacksmith & Plumbing & Entrepreneur & \\
\hline Nurse & Tax accountant & Station attendant & Esthetician & Policeman & Shipbuilder & Quarry & Convenience store & \\
\hline
\end{tabular}

variable of the regression. $x_{i j}$ is a scalar and the value of the $j$-th dimension of occupation $i,\left\|x_{i}\right\|=1$. $\boldsymbol{w}$ is a 300 -dimensional vector. It is the weight for each of the 300 -dimension of $x_{i} . w x_{i}$ is calculated as the dot product between the 300-dimensional vectors $w$ and $x_{i}$. $\alpha_{\text {participant }[i]}$ is the varying intercepts for the participants. That is, $\alpha_{\text {participant }[i]}$ is generated from a normal distribution with a mean of $\alpha_{\text {population }}$ (i.e., a population average) and standard deviation, allowing each participant to have different values in intercepts. The errors $\varepsilon_{i}$ and $\eta_{\text {participant }[i]}$ have independent normal distributions with a mean of zero and standard deviation. To avoid overfitting, we used a prior distribution called the horseshoe prior [5] for $w$ as follows.

$$
\begin{gathered}
w_{j} \sim \operatorname{Normal}\left(0, \lambda_{j} \tau\right), \\
\lambda_{j} \sim \operatorname{Student} t^{+}(3,0,1), \\
\tau \sim \text { Student } t^{+}(2,0,1)
\end{gathered}
$$

Student $t^{+}(3,0,1)$ is a half-Student's t-distribution. We used [21] as reference for the implementation of the horseshoe prior in Rstan. We used the half-Student's t-distribution instead of the half-Cauchy distribution-usually used in the horseshoe prior to allow the parameter estimation to converge.

We conducted a model comparison to test the performance of the regression model using word vectors in terms of prediction. Although it is difficult to set up a benchmark model with a high degree of objective validity, we asked each participant three questions that were highly related to the resource distribution judgment. We then created a benchmark multiple regression model using these three questions. The three questions were as follows. (1) "To what extent do you think each occupation is necessary for the society?" (2) "To what extent do you have a personal need for each of these occupations?" (3) "How much do you think you know about the work of each occupation?" In other words, these questions indicated the social importance, personal importance, and familiarity of each occupation, respectively. Participants answered these questions after completing the vaccination priority judgment for each of the five occupations. The equation of the benchmark regression model is given by:

$$
\begin{aligned}
& y_{i}=\gamma_{\text {participant }[i]} \\
& \quad+\beta_{1} \text { Social importance }_{i}+\beta_{2} \text { Personal importance } i \\
& \\
& \quad+\beta_{3} \text { Famiriality }_{i}+\varepsilon_{i}, \\
& \gamma_{\text {participant }[i]}=\gamma_{\text {population }}+\delta_{\text {participant }[i]}
\end{aligned}
$$

$\gamma_{\text {participant }[i]}$ is the varying intercept for participants. Errors $\varepsilon_{i}$ and $\delta_{\text {participant }[i]}$ have independent normal distributions with a mean of 0 and standard deviation.

We calculated the leave-one-occupation-out correlation (LOOOcorrelation) between the observed and predicted values. That is, we predicted the rating for the hold-out occupation after we trained our model using the other 131 occupations. The model comparison was based on the WAIC. In addition to chiVe, Wikipedia Entity Vectors [24], which uses all the Japanese Wikipedia articles as a training corpus, was used as another pre-trained word2vec resource. We used this pre-trained resource to verify if we could reproduce the results of our model comparison with word vectors trained using a different corpus. We used Rstan version 2.21.2 [23] with $\mathrm{R}$ version 4.1 .0 [19] to fit the regression models. We used the LOO package [25] to estimate the WAIC for model comparison. $\hat{R}$ statistics were below 1.1 for all models, indicating convergence of model estimations. Correlation analyses were performed using JASP 0.14.1 [12]. We used the sklearn version 0.24.1 [18] for k-means clustering. Data and code are available at https: //osf.io/mgpws/?view_only=16acfb4e385e4729b1239c8b8c60ddef

\section{RESULTS}

\subsection{Basic results}

We first checked the basic results regarding the vaccination priority judgment (a 5-point Likert scale ranging from 0 to 4 , with a midpoint of 2). The mean response value was $2.03(S D=1.06, N$ $=243 * 5=1215$ observations). The five occupations that had the highest priority were "doctor" (mean rating $=3.80$ ), "nurse" $(3.70)$, "caregiver" (3.67), "firefighter" (3.43), and "care manager" (3.42). Conversely, the occupations with the lowest five ratings were "painter 
A

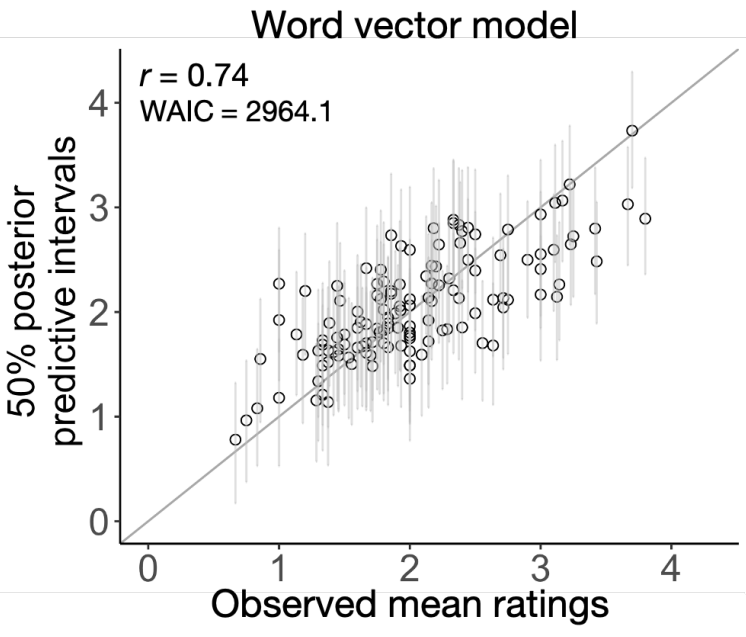

B

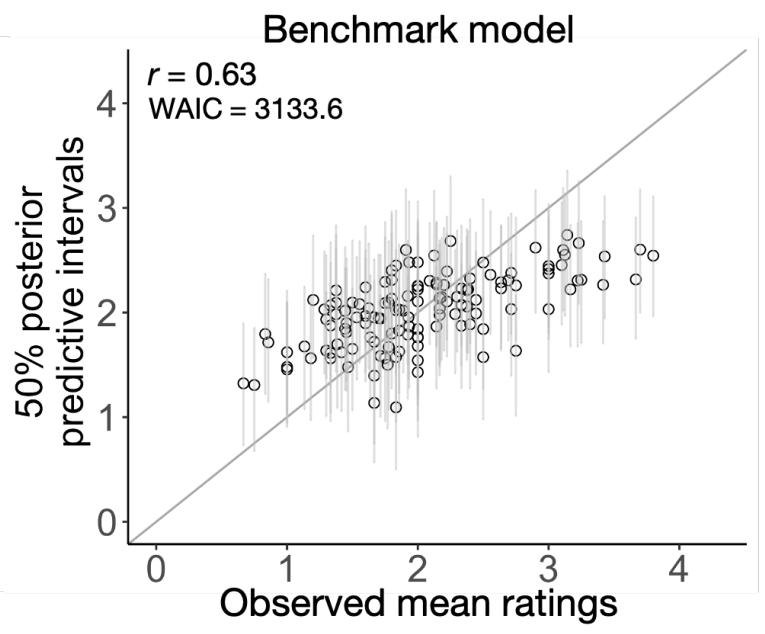

Figure 1: Comparing observed mean ratings for each occupation against predicted values from the word vector model (panel A) and the benchmark model (panel B). The Pearson correlations (r) are leave-one-occupation-out correlations. Each dot represents each of 132 occupations. Error bars indicate the mean $50 \%$ posterior predictive intervals.

(artist)" (0.67), "landlord" (0.75), "illustrator" (0.83), "writer" (0.86), "idol" (i.e., pop star; 1.00), "YouTuber" (1.0), and "fortune teller" (1.0).

\subsection{Predictive accuracy of the word vector model and the benchmark model}

Next, we used the LOOO-correlation and WAIC to check the extent to which the regression model with the word vectors could predict people's vaccination priority ratings. Figure 1A shows the relationship between the observed response (the average rating given by participants to each occupation) and the predicted response by the model (the average rating given by the model to each occupation). Each of the 132 data points in the figure represents an occupation. The observed and predicted responses were matched for the data points on the diagonal. The LOOO-correlation between the observed and predicted responses was $\mathrm{r}=0.74(95 \%$ credible interval $[0.64,0.84])$. The WAIC was $2964.1(\mathrm{SE}=47.3)$. These results confirmed our first prediction that the word vector model would achieve a high out-of-sample correlation $(\mathrm{r}>0.5)$ between the observed and predicted responses. Next, to evaluate the effectiveness of the predictive power of the regression model using the word vectors (i.e., WAIC $=2964.1$ ), we compared the predictive performance of the word vector model with that of the benchmark model. The benchmark model was a regression model consisting of three variables assumed to be closely related to the rating of the vaccination priority judgment. The three variables were (1) social importance, (2) personal importance, and (3) familiarity as rated by the participants for each of the five occupations. Figure $1 \mathrm{~B}$ shows the relationship between the responses predicted by this benchmark model and the observed responses. The correlation (LOOO-correlation) between the observed and predicted responses was $\mathrm{r}=0.63$ [95\% CI $(0.51,0.72)]$. WAIC was $3133.6(\mathrm{SE}=48.9)$. In terms of WAIC (2964.1 vs. 3133.6), we confirmed our prediction that the model using the word vector of the occupations would show better predictive power than the benchmark model.

Furthermore, we confirmed that we could reproduce the results of the model comparison using the WAIC with word vectors obtained from a different corpus. We used Wikipedia Entity Vectors [24], which trained word vectors using the full text of Japanese Wikipedia articles. This pre-trained resource contained 119 of the 132 occupations used for the main analyses. Using only the data for these 119 occupations ( $\mathrm{N}=1103$ observations), we found that the WAIC of the word vector model obtained from Wikipedia Entity word2vec resource was $2719.1(\mathrm{SE}=44.6)$. On the other hand, the WAIC of the benchmark model was 2873.2 ( $\mathrm{SE}=46.0$; we only estimated the WAIC rather than LOOO-correlation because the estimation of LOOO-correlation was computationally expensive). These results indicated that the word vector model performs better than the benchmark model, even when using different vector representations to quantify occupations.

\subsection{Analyses of error rates}

As exploratory analyses, we analyzed leave-one-occupation-out errors (LOOO-errors) for the 132 occupations in both the word vector model and the benchmark model. We defined the LOOO-errors for each occupation as the squared difference between the mean observed response (the average rating given by participants to an occupation) and the mean predicted response by the model (the average rating given by the model to an occupation). Here, again, prediction by the model was performed for the hold-out occupation after we trained the model using the other 131 occupations (leave-one-occupation-out cross-validation). By comparing LOOOerrors for each occupation between the two models, we checked whether the judgment targets that both the word vector model and the benchmark model failed to predict were the same. As shown 


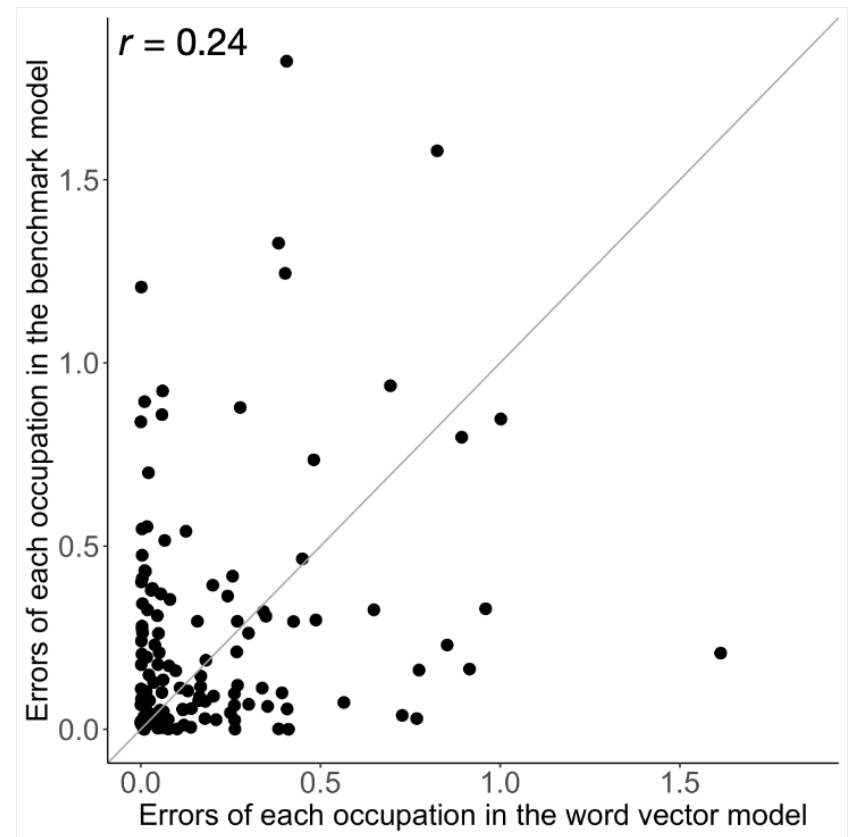

Figure 2: Comparing LOOO-errors between the word vector model and the benchmark model. Each dot represents each occupation, resulting in 132 dots on the plot. Dots above the diagonal indicate occupations that the word vector model predicted better than the benchmark model.

in Figure 2, the correlation of the 132 LOOO-errors between the two models was $r=0.24$ [95\% CI $(0.07,0.39)$ ], which is not considered very high. In fact, in the word vector model, ten judgment targets with the largest LOOO-errors were "fortune teller," "shoe store," "railroad worker," "scientist," "fireman," "YouTuber," "doctor," "janitor," "dry cleaner," and "baker." However, of these ten occupations, only "doctor" appeared in the ten largest LOOO-errors of the benchmark model. These results suggest that there is a great deal of room to investigate the conditions under which prediction by the word vector models can be successful.

\subsection{Interpretation of estimated weights of word vector model}

Finally, following a previous study [28], we investigated the psychological basis of people's judgments on vaccination priority. The coefficient (i.e., weight) of the regression model using word vectors $\boldsymbol{w}$ can itself be considered a 300-dimensional vector. In our model, a word vector with a large dot product between $\boldsymbol{w}$ was associated with high-priority judgments. In contrast, a word vector with a small dot product between $\boldsymbol{w}$ was related to low-priority judgments. For example, the dot product between "nurse vector" and $\boldsymbol{w}$ was 1.82 , resulting in a high priority judgment. While the dot product of "YouTuber vector" and $\boldsymbol{w}$ was -0.69 . Most importantly, we could compute the dot products between $\boldsymbol{w}$ and the vectors of any word in a corpus other than the vectors of occupational words. This meant that we could examine the words or concepts psychologically associated with vaccination priority judgment.
From the frequency list of the Balanced Corpus of Contemporary Written Japanese (BCCWJ, long unit, version 1.0) provided by the National Institute for Japanese Language and Linguistics, we selected 5000 most frequently used words after excluding stop words such as particles, auxiliary verbs, adverbs, conjunctions, interjections, pronouns, adnominal adjectives, and prefixes. After removing duplicate words, we narrowed our selection to words included in the pre-trained word2vec resource (chiVe) we used for our main analyses, resulting in 4,196 unique words. We then calculated the dot product of $\boldsymbol{w}$ and each vector of the 4,196 words, resulting in 4.196 dot products. Note that the magnitude of the word vectors of 4,196 words was normalized to one before calculating the dot products.

The 200 words that had the highest and the lowest dot product with $\boldsymbol{w}$ were each subjected to k-means clustering. We set the number of clusters to 10 . Table 2 shows the results (for space limitations, we show five words for each cluster). Table $2 \mathrm{~A}$ shows that concepts related to medicine (Cluster 2), public transportation (Cluster 5), childcare service (Cluster 6), and administrative service (Cluster 8) might be related to the judgment dimension for most prioritized occupations. In contrast, Table $2 \mathrm{~B}$ shows concepts related to money (Cluster 1), outside (Cluster 3), and literature (Cluster 4) may have been related to the dimension of least prioritized occupations. These results provide insights into the psychological dimensions underlying the participants' vaccination priority judgments.

\section{DISCUSSION}

The allocation of limited resources is a crucial social decision. In this study, we focused on the fact that many human societies consist of a high degree of division of labor based on occupations and investigated the resource allocation judgment about occupations. Specifically, we attempted to predict how people made priority judgments when distributing scarce resources across various occupations and explored the psychological basis of these judgments. A unique feature of this study was that we used vector representations of occupations obtained from a large corpus to quantify the meanings of various occupations.

The analysis showed that the regression model using the word vectors of occupations could predict people's vaccination priority judgment better than the benchmark model set up in this study. We also analyzed the errors for each of the 132 occupations in the word vector and benchmark models. We found that there was not much overlap in the occupations that could not be predicted by either model. Although further investigation is needed, this result suggested that there might be positive complementary aspects to the model using corpus data (word vector model) and the model using participants' responses (benchmark model). Finally, we explored the psychological bases of people's judgments, using $\boldsymbol{w}$-the weight parameter of the word vector model. As a result, we found that concepts related to medicine and public transportation may be associated with the judgment dimension for most prioritized occupations. In contrast, concepts related to money and literature may be related to the dimension of least prioritized occupations. In summary, this study showed that the word vectors representing people's knowledge representation about more than 130 different 
Table 2: Results of k-means clustering. Table A shows the results of k-means clustering for words that had the 200highest dot products with $w$. We set the number of clusters as 10. For space limitations, we showed five words for each cluster. Table B represents results from the 200lowest words in the same way as Table A. The word "doctor (isha)" is different from the word "doctor (ishi)," which is one of the 132 occupations. For corresponding Japanese words, see Table S2 provided in https://osf.io/mgpws/?view only=16acfb4e385e4729b1239c8b8c60ddef

\begin{tabular}{|c|c|c|c|c|}
\hline \multicolumn{5}{|l|}{$\mathbf{A}$} \\
\hline 1 Staff & Responsible person & Users & Capacity & Workplace \\
\hline 2 Medical institution & Hospital & Medicine & Patient & Doctor (isha) \\
\hline 3 Medical Care & Nursing Care & Welfare & Medical cost & Disaster \\
\hline $4 \begin{array}{l}\text { Ministry of Health, } \\
\text { Labour and Welfare }\end{array}$ & Administration & Authorization & Local government & Municipality \\
\hline 5 Passenger & Airport & Driver & Trafic & Cab \\
\hline 6 Nursery school & Animals & Birth & Childcare & Kindergarten \\
\hline 7 Day off & Time of day & Numberofpeople & Holiday & Morning \\
\hline 8 Civil servant & Government office & $\begin{array}{l}\text { Self- } \\
\text { defenseforce }\end{array}$ & Police & Section chief \\
\hline 9 City hall & Mail & Postoffice & Cigarette & Hotel \\
\hline 10 Obvious & Reduction & Aging & Increase & Augment \\
\hline
\end{tabular}

B

\begin{tabular}{|c|c|c|c|c|}
\hline 1 Accumulate & Gold & Polish & Commerce & Cards \\
\hline 2 Advantage & Good & Control & Superior & Chance \\
\hline 3 Ground & Rock & Soil & Trees & Water \\
\hline 4 Painting & Text & Phrase & Sentence & Expression \\
\hline 5 Stock (Kabushiki) & Loan & Stock (Kabu) & Interest & Owner \\
\hline 6 Land & Mansion & Meiji & Merchant & Alley \\
\hline 7 Hue & Release & Skillful & Color & Rose \\
\hline 8 Dialogue & Hint & Conversation & Technique & Kanji \\
\hline 9 Unique & Expensive & Original & Explanation & Authentic \\
\hline 10 Held & Homepage & Establishment & Hiroshima & Various places \\
\hline
\end{tabular}

occupations can help predict and understand their distributional judgments.

One of the important features of the present research was the pursuit of both prediction and understanding. For pursuing predictive performance, models such as deep neural networks were better options; however, there are some difficulties in interpreting complex models [22]. At the same time, the use of these types of complex models usually requires large amounts of data. Thus, we assumed that directly applying these types of models was not suitable for the present study.

However, if the main goal was interpretation, we could have used the same approach as the benchmark models in this study. For example, we could examine whether the social importance of each occupation would be positively related to the ratings of vaccination priority judgments. This might have helped us interpret the relationship between each occupation's social importance and priority in vaccination. However, this approach has the disadvantage of inadequately quantifying the diverse knowledge representations and connotations associated with each occupation, resulting in lower predictive performance. Our modeling approach using word vectors showed adequately high out-of-sample predictive accuracy by quantifying the knowledge representation of each occupation based on the distribution patterns of occupational concepts in a large corpus. In addition, unlike other studies using high predictive power but too complex models, the analysis using the word vectors in this study was only performed in the framework of multiple regression analysis, which allowed for a straightforward interpretation of the results. Recent research in social science has examined the importance of not only explaining or understanding, which has been the focus of social science in the past, but also focusing on prediction, which has been the focus of the machine learning community [11, 26, 27]. This study showed that the word vector model was an efficient way to achieve the right balance of both prediction and understanding.

While we conducted the current study in May 2021, the word vectors used for the main analyses were obtained from a corpus from 2011-2015. This did not reflect potential changes in semantic space caused by COVID-19, a large-scale societal-level phenomenon occurring around early 2020. Nonetheless, our model using the word vectors for each occupation showed relatively good predictive accuracy in our setting, suggesting that the word-vector modeling may be a robust approach for semantic space changes. Of course, if an event that could completely change the correlation structure of people's knowledge representation of occupations occurs (e.g., the industrial revolution), it will be necessary to acquire word vectors from a corpus created after the event occurred. Future research can systematically investigate how the use of knowledge representations from multiple corpora created at different times affects the prediction of people's decisions and judgments.

One clear limitation of using word vectors to predict people's judgments was that this approach was, currently, not well suited for predicting individuals' judgments and decision-making. The pre-trained word2vec resource employed in this study used many web pages to acquire vector representations of the words, causing a given occupational vector to reflect the average knowledge representation of many people (i.e., the diverse texts in the corpus) for that occupation; heterogeneity of each participant's knowledge representation for a given occupation could not be considered. Therefore, an approach such as the present one may not be sufficient for predicting the judgments of specific individuals with high accuracy. For example, the semantic impression of the younger and the older generation towards occupations could differ, but the current study cannot take this heterogeneity into account. This problem can be solved using a corpus that reflects the knowledge representation of a particular individual. However, such corpus is usually not accessible, and even if it is, privacy issues may arise. Research on finer-scale predictions using word vectors is both challenging and promising in the future.

We also acknowledge that there is still much to be clarified regarding the usefulness of the word vector model. To determine how well the word vector model performs, we conducted a model comparison using the benchmark regression analysis of three explanatory variables associated with the resource distribution judgment. Then, we showed that the model using word vectors was superior to the benchmark model in terms of the WAIC. However, we agree that there are many other possible ways to set up a benchmark model. More importantly, the usefulness of a model should ultimately be evaluated by the benefits it brings to the real world. 
Therefore, in the future, it will be essential to conduct a more objective evaluation of the applicability and usefulness of the model in the real world.

Modeling distributional judgments for everyday concepts, such as occupation, has been difficult in the past. However, large naturalistic language data have made it possible to measure the meanings of various concepts and objects [16]. The present study used knowledge representations of occupations quantified from word distributions in a large corpus to predict and understand people's distributional judgments for more than 130 different occupations. Although such an approach is still in its infancy, we believe that modeling judgments based on the meanings of everyday concepts quantified by word vectors is a promising way for social sciences, including psychology, cognitive science, and cultural sociology.

\section{ACKNOWLEDGMENTS}

This work was funded by JSPS KAKENHI grants (nos. JP20H01563 and JP21J00403).

\section{REFERENCES}

[1] Stacy J. Adams. 1963. Towards an understanding of inequity. The Journal of Abnormal and Social Psychology 67, 5 (May 1963), 422-436. DOI:https://doi.org/ $10.1037 / \mathrm{h} 0040968$

[2] Alberto Alesina and Paola Giuliano. 2009. Preferences for Redistribution. National Bureau of Economic Research, Cambridge, MA. DOI:https://doi.org/10.3386/ w14825

[3] Michael M. Bechtel and Roman Liesch. 2020. Reforms and redistribution: Disentangling the egoistic and sociotropic origins of voter preferences. Public Opinion Quarterly 84, 1 (July 2020), 1-23. DOI:https://doi.org/10.1093/poq/nfaa006

[4] Sudeep Bhatia. 2019. Predicting risk perception: New insights from data science. Management Science 65, 8 (August 2019), 3800-3823. DOI:https://doi.org/10.1287/ mnsc.2018.3121

[5] Carlos M. Carvalho, Nicholas G. Polson, and James G. Scott. 2010. The horseshoe estimator for sparse signals. Biometrika 97, 2 (June 2010), 465-480. DOI:https: //doi.org/10.1093/biomet/asq017

[6] Garry Charness and Matthew Rabin. 2002. Understanding social preferences with simple tests. The Quarterly Journal of Economics 117, 3 (August 2002), 817-869. DOI:https://doi.org/10.1162/003355302760193904

[7] Dirk Engelmann and Martin Strobel. 2004. Inequality aversion, efficiency, and maximin preferences in simple distribution experiments. American Economic Review 94, 4 (August 2004), 857-869. DOI:https://doi.org/10.1257/0002828042002741

[8] Ernst Fehr and Klaus M. Schmidt. 1999. A theory of fairness, competition, and cooperation. The Quarterly Journal of Economics 114, 3 (August 1999), 817-868. DOI:https://doi.org/10.1162/003355399556151

[9] Vael Gates, Thomas L. Griffiths, and Anca D. Dragan. 2020. How to be helpful to multiple people at once. Cogn. Sci. 44, 6 (June 2020), e12841. DOI:https://doi.org/ 10.1111/cogs. 12841

[10] Zellig S. Harris. 1954. Distributional Structure. WORD 10, 2-3 (August 1954), 146-162. DOI:https://doi.org/10.1080/00437956.1954.11659520

[11] Jake M. Hofman, Duncan J. Watts, Susan Athey, Filiz Garip, Thomas L. Griffiths, Jon Kleinberg, Helen Margetts, Sendhil Mullainathan, Matthew J. Salganik, Simine Vazire, Alessandro Vespignani, and Tal Yarkoni. 2021. Integrating explanation and prediction in computational social science. Nature 595, 7866 (July 2021), 181-188. DOI:https://doi.org/10.1038/s41586-021-03659-0

[12] JASP Team. 2020. JASP (Version 0.14.1)[Computer software]. Retrieved from https://jasp-stats.org/

[13] Tatsuya Kameda, Keigo Inukai, Satomi Higuchi, Akitoshi Ogawa, Hackjin Kim, Tetsuya Matsuda, and Masamichi Sakagami. 2016. Rawlsian maximin rule operates as a common cognitive anchor in distributive justice and risky decisions. Proc. Natl. Acad. Sci. USA 113, 42 (October 2016), 11817-11822. DOI:https: //doi.org/10.1073/pnas.1602641113

[14] Hitoshi Manabe, Teruaki Oka, Yoshitaka Umikawa, Kazuma Takaoka, Yoshitaka Uchida, and Masayuki Asahara. 2019. Japanese word embedding based on multigranular tokenization results (in Japanese). In Proceedings of the Twenty-fifth Annual Meeting of the Association for Natural Language Processing (NLP2019), March 13-15, 2019, Nagoya, Japan. The Association for Natural Language Processing, Kyoto, Japan, NLP2019-P8-5.

[15] Tomas Mikolov, Kai Chen, Greg Corrado, and Jeffrey Dean. 2013. Efficient estimation of word representations in vector space. arXiv:1301.3781 [cs]. Retrieved from http://arxiv.org/abs/1301.3781

[16] John Mohr, Christopher Bail, Margaret Frye, Jennifer Lena, and Omar Lizardo. 2020. Measuring culture. Columbia University Press, New York, NY.

[17] Michael I. Norton and Dan Ariely. 2011. Building a Better America-One Wealth Quintile at a Time. Perspect. Psychol. Sci. 6, 1 (January 2011), 9-12. DOI:https: //doi.org/10.1177/1745691610393524

[18] Fabian Pedregosa, Gael Varoquaux, Alexandre Gramfort, Vincent Michel, Bertrand Thirion, Olivier Grisel, Mathieu Blondel, Peter Prettenhofer, Ron Weiss, Vincent Dubourg, Jake Vanderplas, Alexandre Passos, David Cournapeau, Matthieu Brucher, Matthieu Perrot, and Edouard Duchesnay. 2011. Scikit-learn: Machine learning in Python. Journal of Machine Learning Research 12, 85 (October 2011), 2825-2830.

[19] R Core Team. 2021. R: A language and environment for statistical computing. R Foundation for Statistical Computing, Vienna, Austria. Retrieved from https: //www.R-project.org/

[20] Russell Richie, Wanling Zou, and Sudeep Bhatia. 2019. Predicting high-level human judgment across diverse behavioral domains. Collabra: Psychology 5, 1 (January 2019), 50. DOI:https://doi.org/10.1525/collabra.282

[21] Allen Riddell. 2014. Epistemology of the corral: Regression and variable selection with Stan and the Horseshoe prior. Retrieved August 6, 2021 from https://ariddell. org/horseshoe-prior-with-stan.html

[22] Cynthia Rudin. 2019. Stop explaining black box machine learning models for high stakes decisions and use interpretable models instead. Nat. Mach. Intell. 1, 5 (May 2019), 206-215. DOI:https://doi.org/10.1038/s42256-019-0048-x

[23] Stan Development Team. 2020. RStan: the R interface to Stan. Retrieved from http://mc-stan.org/

[24] Masatoshi Suzuki, Koji Matsuda, Satoshi Sekine, Naoaki Okazaki, and Kentaro Inui. 2018. A joint neural model for fine-grained named entity classification of Wikipedia articles. IEICE Trans. Inf. \& Syst. E101.D, 1 (2018), 73-81. DOI:https: //doi.org/10.1587/transinf.2017SWP0005

[25] Aki Vehtari, Jonah Gabry, Mans Magnusson, Yuling Yao, Paul-Christian Bürkner, Topi Paananen, and Andrew Gelman. 2020. loo: Efficient leave-one-out crossvalidation and WAIC for Bayesian models. Retrieved from https://mc-stan.org/ loo/

[26] Duncan J. Watts. 2014. Common sense and sociological explanations. American Journal of Sociology 120, 2 (September 2014), 313-351. DOI:https://doi.org/10. $1086 / 678271$

[27] Tal Yarkoni and Jacob Westfall. 2017. Choosing prediction over explanation in psychology: Lessons from machine learning. Perspect. Psychol. Sci. 12, 6 (November 2017), 1100-1122. DOI:https://doi.org/10.1177/1745691617693393

[28] Wanling Zou and Sudeep Bhatia. 2021. Judgment errors in naturalistic numerical estimation. Cognition 211, (June 2021), 104647. 